\title{
Synthesis and antimicrobal activities of some novel triazolo[1,5-a]pyrimidine derivatives
}

\author{
Sagar P. Gami, Kalpesh V. Vilapara, Hasmukh R. Khunt, Jayesh S. Babariya, \\ Yogesh T. Naliapara* \\ Department of Chemistry, Saurashtra University, Rajkot-36005, Gujarat, India \\ *E-mail address: naliaparachem@yahoo.co.in
}

\begin{abstract}
A convenient synthesis of substituted 1,2,4-triazolo[1,5-a]pyrimidine was carried out by the reaction of various ketene dithioacetals with 5-amino 1,2,4-triazole in methanol in presence of sodium methoxide. The newly synthesized compound were characterized by $1 \mathrm{H} N \mathrm{NR},{ }^{13} \mathrm{C} N \mathrm{NMR}, \mathrm{IR}, \mathrm{MS}$, elemental analysis and screened for their antimicrobial activity against various strains of bacteria and fungi.
\end{abstract}

Keywords: 5-amino-1,2,4-triazole; ketene dithioacetal; antimicrobial activity; triazolopyrimidine

\section{INTRODUCTION}

Various fused pyrimidines like triazolopyrimidines, pyrazolopyrimidines, pyrimidoazepines, pteridines, pyridopyrimidines, purines, quinazolines, furopyrimidines, and pyrrolopyrimidines were studied in the past decade and were found to possess remarkable pharmacological properties. Triazolopyrimidines are new class of hybrid heterocycles of pyrimidine ring fused with triazole and possessing improved activity. The condensation of pyrimidine with triazole ring gives bicyclic heterocycles known as 1,2,4-triazolopyrimidines, which exist in four isomeric structure. ${ }^{[1-4]}$ Among these four structural isomers, 1,2,4triazolo[1,5-a]pyrimidine derivatives are thermodynamically more stable and most studied ones.

Fused hetero aromatic systems are often of much greater interest in biological activity than the constituent monocyclic compounds. 1,2,4-triazolo[1,5-a]pyrimidines have diverse pharmacological activities, such as antitumor potency ${ }^{[5-8]}$, antimalarial ${ }^{[9]}$, antimicrobial ${ }^{[10-13]}$, anti-inflammatory $^{[14]}$ inhibition of KDR kinase ${ }^{[15]}$, antifungal ${ }^{[16]}$ and macrophage activation $^{[17]}$. They have proved to be promising anticancer ${ }^{[18]}$ agents with dual mechanisms of tubulin polymerization promotion ${ }^{[5,6]}$ as well as cyclin dependent kinases 2 inhibition ${ }^{[19]}$.

We have synthesize 1,2,4-triazolo[1,5-a]pyrimidine derivatives by refluxing various ketene dithioacetals with 5-amino-1,2,4-triazole in the presence of sodium methoxide in methanol ${ }^{[20-22]}$. The newly synthesized compounds were characterized by IR, Mass, $1 \mathrm{H}$ NMR, ${ }^{13} \mathrm{C}$ NMR spectroscopy and elemental analysis. All the synthesized compounds were evaluated for their antimicrobial activity. 


\section{EXPERIMENTAL}

Thin-layer chromatography was accomplished on $0.2 \mathrm{~mm}$ precoated plates of silica gel G60 F254 (Merck). Visualization was made with UV light (254 and $365 \mathrm{~nm}$ ) or with an iodine vapor. IR spectra were recorded on a FTIR-8400 spectrophotometer using DRS prob. ${ }^{1} \mathrm{H}(400 \mathrm{MHz}),{ }^{13} \mathrm{C}(100 \mathrm{MHz}) \mathrm{NMR}$ spectra were recorded on a Bruker AVANCE II spectrometer in $\mathrm{CDCl}_{3}$ and DMSO. Chemical shifts are expressed in $\delta$ ppm downfield from TMS as an internal standard. Mass spectra were determined using direct inlet probe on a GCMS-QP 2010 mass spectrometer (Shimadzu). Solvents were evaporated with a BUCHI rotary evaporator. Melting points were measured in open capillaries and are uncorrected.

General synthesis of 4-methyl-3-oxo-N-arylpentanamide (Int 1a-t); A mixture of aromatic amine $(10 \mathrm{mmol})$, methyl 4-methyl-3-oxopentanoate $(10 \mathrm{mmol})$ and catalytic amount of sodium or potassium hydroxide lie $(10 \%)$ in toluene $(50 \mathrm{ml})$ was refluxed at $110{ }^{\circ} \mathrm{C}$ for $12-15$ $\mathrm{h}$. The reaction was monitored by TLC. After completion of reaction, the solvent was removed under reduce pressure and washed with water to afford pure product.

General synthesis of ketene dithioacetals (Int 2a-t); To a well stirred suspension of 4methyl-3-oxo-N-arylpentanamide $(10 \mathrm{mmol})$ and potassium carbonate $(20 \mathrm{mmol})$ in DMF (20 $\mathrm{mL})$ at $0-5{ }^{\circ} \mathrm{C}$ was added $\mathrm{CS}_{2}(10 \mathrm{mmol})$ over a period of $30 \mathrm{~min}$. After completion of the addition, the reaction mixture was stirred at $0-5^{\circ} \mathrm{C}$ for $1 \mathrm{~h}$. Appearance of reddish solid in the reaction medium indicated the formation of dipotassium salt. To this reaction, a solution of methyl iodide $(20 \mathrm{mmol})$ was added drop wise within $15 \mathrm{~min}$ at $0-5{ }^{\circ} \mathrm{C}$. The mixture was allowed to warm at room temperature and stirred for $15 \mathrm{~h}$, and then poured onto crushed ice under stirring. The separated solid was washed with water and collected by filtration.

General synthesis of triazolopyrimidine (3a-t); To a solution of sodium methoxide in methanol $(10 \mathrm{~mL})$ ketene dithioacetal $(5 \mathrm{mmol})$ and 5-amino-1,2,4-triazole were added at 0-5 ${ }^{\circ} \mathrm{C}$. The reaction mixture then refluxed for 2-4 hours. The reaction was monitored by TLC. After the completion of reaction mixture was poured into cold water and the separated solid was dried and purified by column chromatography using ethylacetate and hexane.

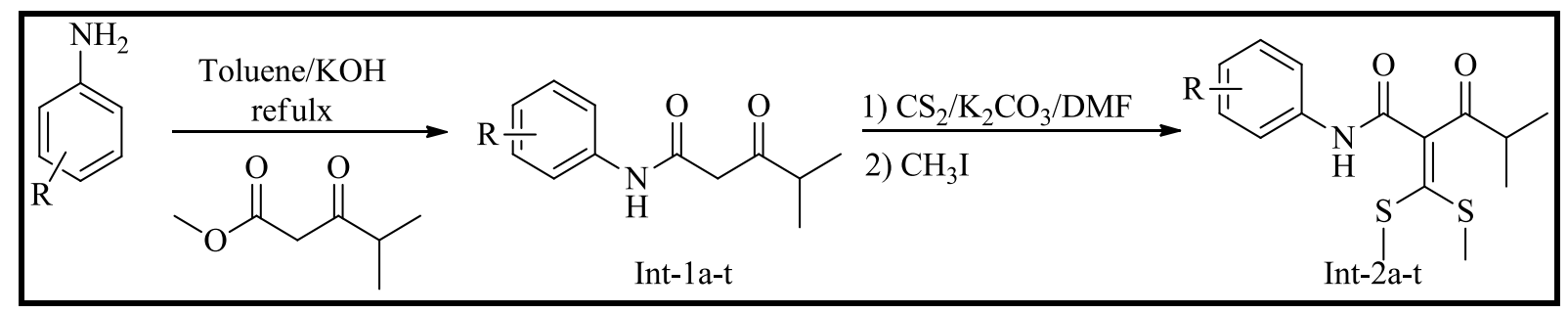

Figure 1. Reaction Scheme for 2a-t. 


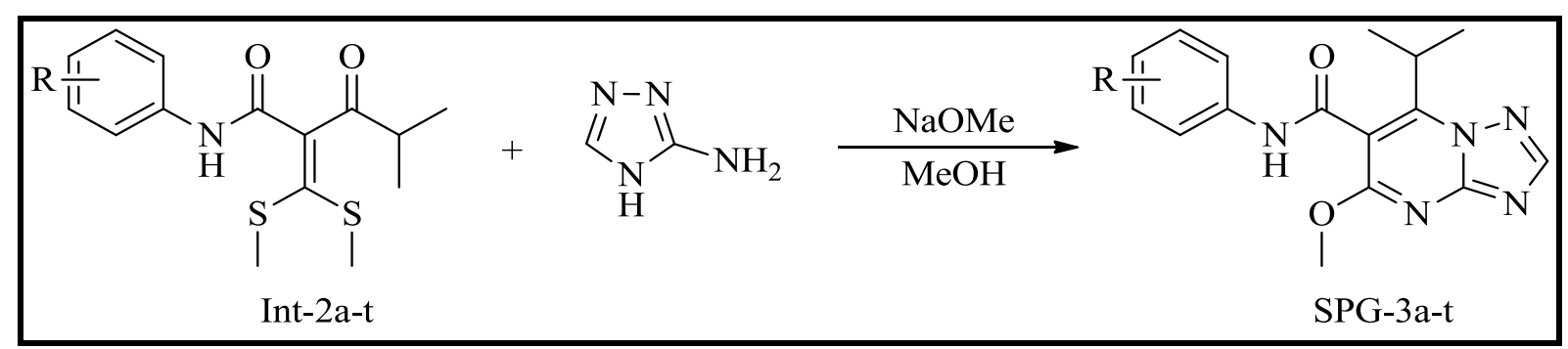

Figure 2. Reaction Scheme for 3a-t.

N-(3-chloro-4-fluorophenyl)-7-isopropyl-5-methoxy-[1,2,4]triazolo[1,5-a]pyrimidine-6carboxamide (SPG-3h): White solid; $\mathrm{mp} 256-258{ }^{\circ} \mathrm{C}$; Rf 0.45 (4:6 hexane-EtOAc); IR (KBr): 3244, 3198, 3124, 3055, 2933, 2868, 1680, 1622, 1539, 1394, 1336, 1271, 1205, 1124 , 1020, 958, 827, 756, 665, $567 \mathrm{~cm}-1$; $1 \mathrm{H}$ NMR: $\left.\delta 1.480-1.1 .497\left(\mathrm{~d}, 6 \mathrm{H},\left(\mathrm{CH}_{3}\right)_{2}\right), j=6.8 \mathrm{~Hz}\right)$, 3.506-3.540 (m, 1H, -CH), $4.041(\mathrm{~s}, 3 \mathrm{H},-\mathrm{OCH} 3), 7.434-7.479(\mathrm{t}, 1 \mathrm{H}, \mathrm{Ar}-\mathrm{H}), 7.522(\mathrm{~s}, 1 \mathrm{H}$, Ar-H), 8.010-8.022 (d, 1H, Ar-H, $j=4.8 \mathrm{~Hz}), 8.575$ (s, 1H, $-\mathrm{CH}$ triazole ring), $10.951(\mathrm{~s}, 1 \mathrm{H}$, -CONH); 13C NMR (100 MHz, DMSO): 18.07, 30.56, 55.06, 110.19, 117.14, 119.33, $119.56,120.61,135.57,152.35,152.95,153.90,154.78,160.84,162.52 ; \mathrm{MS}(\mathrm{m} / \mathrm{z}): 363$ $(\mathrm{M}+)$; Anal. Calcd for $\mathrm{C}_{16} \mathrm{H}_{15} \mathrm{ClFN}_{5} \mathrm{O}_{2}:$ C, 52.83; H, 4.16; N, 19.25; Found: C, 52.78; H, 4.21; N, 19.18 .

N-(3,4-dimethylphenyl)-7-isopropyl-5-methoxy-[1,2,4]triazolo[1,5-a]pyrimidine-6-

carboxamide (SPG-3k): Pale yellow solid; $\mathrm{mp} 224-226{ }^{\circ} \mathrm{C} ; \mathrm{R}_{\mathrm{f}} 0.44$ (4:6 hexane-EtOAc); IR (KBr): 3245, 3196, 3117, 3059, 2924, 2858, 1730, 1678, 1616, 1535, 1456, 1396, 1278, 1205 , 1124, 1020, 958, 827, 756, 665, $567 \mathrm{~cm}^{-1}$; ${ }^{1} \mathrm{H}$ NMR: $\delta$ 1.476-1.1.492 (d, 6H, $\left.\left(\mathrm{CH}_{3}\right)_{2}\right), j=6.4$ $\mathrm{Hz}), 2.198\left(\mathrm{~s}, 3 \mathrm{H},-\mathrm{CH}_{3}\right), 2.221\left(\mathrm{~s}, 3 \mathrm{H},-\mathrm{CH}_{3}\right), 3.498-3.532(\mathrm{~m}, 1 \mathrm{H},-\mathrm{CH}), 4.029(\mathrm{~s}, 3 \mathrm{H},-$ $\left.\mathrm{OCH}_{3}\right), 7.111-7.130(\mathrm{~d}, 1 \mathrm{H}, \mathrm{Ar}-\mathrm{H}, j=7.6 \mathrm{~Hz}), 7.368-7.387(\mathrm{~d}, 1 \mathrm{H}, \mathrm{Ar}-\mathrm{H}, j=7.6 \mathrm{~Hz}), 7.453$ $(\mathrm{s}, 1 \mathrm{H}, \mathrm{Ar}-\mathrm{H}), 8.552\left(\mathrm{~s}, 1 \mathrm{H},-\mathrm{CH}\right.$ triazole ring), $10.504(\mathrm{~s}, 1 \mathrm{H},-\mathrm{CONH}) ;{ }^{13} \mathrm{C}$ NMR $(100 \mathrm{MHz}$, DMSO): 18.04, 18.77, 19.51, 30.51, 54.94, 110.86, 116.64, 120.24, 128.57, 129.66, 131.50, 136.22, 152.52, 153.83, 154.85, 160.24, 162.68, 166.89; MS ( $\mathrm{m} / \mathrm{z}): 339\left(\mathrm{M}^{+}\right)$; Anal. Calcd for $\mathrm{C}_{18} \mathrm{H}_{21} \mathrm{~N}_{5} \mathrm{O}_{2}$ : C, 63.70; H, 6.24; N, 20.64; Found: C, 63.68; H, 6.31; N, 20.59.

7-isopropyl-5-methoxy-N-(p-tolyl)-[1,2,4] triazolo[1,5-a]pyrimidine-6-carboxamide (SPG-3m): White solid; $\mathrm{mp} 278-280{ }^{\circ} \mathrm{C}$; $\mathrm{R}_{\mathrm{f}} 0.49$ (4:6 hexane-EtOAc); IR (KBr): 3292, 3115, $3045,2931,1633,1550,1512,1465,1404,1290,1199,989,812,759,659,505 \mathrm{~cm}^{-1} ;{ }^{1} \mathrm{H}$ NMR: $\delta$ 1.476-1.1.494 (d, 6H, $\left.\left.\left(\mathrm{CH}_{3}\right)_{2}\right), j=7.2 \mathrm{~Hz}\right), 2.286\left(\mathrm{~s}, 3 \mathrm{H},-\mathrm{CH}_{3}\right), 3.480-3.550(\mathrm{~m}, 1 \mathrm{H}$, $-\mathrm{CH}), 4.031\left(\mathrm{~s}, 3 \mathrm{H},-\mathrm{OCH}_{3}\right), 7.171-7.192(\mathrm{~d}, 2 \mathrm{H}, \mathrm{Ar}-\mathrm{H}, j=8.4 \mathrm{~Hz}), 7.541-7.562(\mathrm{~d}, 2 \mathrm{H}, \mathrm{Ar}-\mathrm{H}$, $j=8.4 \mathrm{~Hz}), 8.556(\mathrm{~s}, 1 \mathrm{H},-\mathrm{CH}$ triazole ring $), 10.589(\mathrm{~s}, 1 \mathrm{H},-\mathrm{CONH}) ;{ }^{13} \mathrm{C} \mathrm{NMR}(100 \mathrm{MHz}$, DMSO): 18.03, 20.40, 30.51, 54.96, 110.77, 119.12, 129.24, 133.13, 135.95, 152.56, 153.82, 154.86, 160.29, 162.65; MS (m/z): $325\left(\mathrm{M}^{+}\right)$; Anal. Calcd for $\mathrm{C}_{17} \mathrm{H}_{19} \mathrm{~N}_{5} \mathrm{O}_{2}: \mathrm{C}, 62.75 ; \mathrm{H}, 5.89$; N, 21.52; Found: C, 62.69; H, 5.93; N, 21.48. 
Table 1. Physical Data of Compound SPG 3a-t.

\begin{tabular}{|c|c|c|c|c|c|}
\hline Compound & $\mathbf{R}$ & M.F. & M.W. & M.P. $\left({ }^{\circ} \mathrm{C}\right)$ & Yield (\%) \\
\hline SPG-3a & $\mathrm{H}$ & $\mathrm{C}_{16} \mathrm{H}_{17} \mathrm{~N}_{5} \mathrm{O}_{2}$ & 311 & $236-238$ & 40 \\
\hline SPG-3b & $4-\mathrm{Br}$ & $\mathrm{C}_{16} \mathrm{H}_{16} \mathrm{BrN}_{5} \mathrm{O}_{2}$ & 390 & $246-248$ & 36 \\
\hline SPG-3c & $4-\mathrm{F}$ & $\mathrm{C}_{16} \mathrm{H}_{16} \mathrm{FN}_{5} \mathrm{O}_{2}$ & 329 & $224-226$ & 35 \\
\hline SPG-3d & $4-\mathrm{OCH}_{3}$ & $\mathrm{C}_{17} \mathrm{H}_{19} \mathrm{~N}_{5} \mathrm{O}_{3}$ & 341 & $238-240$ & 42 \\
\hline SPG-3e & $4-\mathrm{NO}_{2}$ & $\mathrm{C}_{16} \mathrm{H}_{16} \mathrm{~N}_{6} \mathrm{O}_{4}$ & 341 & $250-252$ & 25 \\
\hline SPG-3f & $3-\mathrm{CH}_{3}$ & $\mathrm{C}_{17} \mathrm{H}_{19} \mathrm{~N}_{5} \mathrm{O}_{2}$ & 325 & $260-262$ & 35 \\
\hline SPG-3g & 3,4-di-Cl & $\mathrm{C}_{16} \mathrm{H}_{15} \mathrm{Cl}_{2} \mathrm{~N}_{5} \mathrm{O}_{2}$ & 380 & $244-246$ & 30 \\
\hline SPG-3h & $3-\mathrm{Cl}, 4-\mathrm{F}$ & $\mathrm{C}_{16} \mathrm{H}_{15} \mathrm{ClFN}_{5} \mathrm{O}_{2}$ & 363 & $256-258$ & 32 \\
\hline SPG-3i & $4-\mathrm{Cl}$ & $\mathrm{C}_{16} \mathrm{H}_{16} \mathrm{ClN}_{5} \mathrm{O}_{2}$ & 345 & $258-260$ & 35 \\
\hline SPG-3j & 2,4-di-Cl & $\mathrm{C}_{16} \mathrm{H}_{15} \mathrm{Cl}_{2} \mathrm{~N}_{5} \mathrm{O}_{2}$ & 380 & $282-284$ & 32 \\
\hline SPG-3k & $3,4-\mathrm{di}-\mathrm{CH}_{3}$ & $\mathrm{C}_{18} \mathrm{H}_{21} \mathrm{~N}_{5} \mathrm{O}_{2}$ & 339 & $224-226$ & 40 \\
\hline SPG-3I & $2-\mathrm{Cl}$ & $\mathrm{C}_{16} \mathrm{H}_{16} \mathrm{ClN}_{5} \mathrm{O}_{2}$ & 345 & $276-278$ & 30 \\
\hline SPG-3m & $4-\mathrm{CH}_{3}$ & $\mathrm{C}_{17} \mathrm{H}_{19} \mathrm{~N}_{5} \mathrm{O}_{2}$ & 325 & $278-280$ & 35 \\
\hline SPG-3n & $2,3-\mathrm{di}-\mathrm{CH}_{3}$ & $\mathrm{C}_{18} \mathrm{H}_{21} \mathrm{~N}_{5} \mathrm{O}_{2}$ & 339 & $268-270$ & 38 \\
\hline SPG-3o & $2-\mathrm{F}$ & $\mathrm{C}_{16} \mathrm{H}_{16} \mathrm{FN}_{5} \mathrm{O}_{2}$ & 329 & $232-234$ & 33 \\
\hline SPG-3p & $2-\mathrm{NO}_{2}$ & $\mathrm{C}_{16} \mathrm{H}_{16} \mathrm{~N}_{6} \mathrm{O}_{4}$ & 356 & $286-288$ & 26 \\
\hline SPG-3q & $3-\mathrm{Cl}$ & $\mathrm{C}_{16} \mathrm{H}_{16} \mathrm{ClN}_{5} \mathrm{O}_{2}$ & 345 & $264-266$ & 35 \\
\hline SPG-3r & $4-\mathrm{Cl}, 2-\mathrm{NO}_{2}$ & $\mathrm{C}_{16} \mathrm{H}_{15} \mathrm{ClN}_{6} \mathrm{O}_{4}$ & 390 & $274-276$ & 22 \\
\hline SPG-3s & $4-\mathrm{Cl}, 3-\mathrm{NO}_{2}$ & $\mathrm{C}_{16} \mathrm{H}_{15} \mathrm{ClN}_{6} \mathrm{O}_{4}$ & 390 & $266-268$ & 25 \\
\hline SPG-3t & $2-\mathrm{CF}_{3}$ & $\mathrm{C}_{17} \mathrm{H}_{16} \mathrm{~F}_{3} \mathrm{~N}_{5} \mathrm{O}_{2}$ & 379 & $234-236$ & 35 \\
\hline
\end{tabular}


Table 2. Antibacterial activity of compound SPG 3a-t.

\begin{tabular}{|c|c|c|c|c|c|}
\hline \multirow{2}{*}{ Sr. No. } & \multirow{2}{*}{ Code } & \multicolumn{4}{|c|}{$\operatorname{MIC}(\mu \mathrm{g} / \mathrm{mL})$} \\
\hline & & E.coli & P.aeruginosa & S.aureus & S.pyogenus \\
\hline 1 & SPG-5a & 500 & 500 & 500 & 500 \\
\hline 2 & SPG-5b & 250 & 250 & 200 & 250 \\
\hline 3 & SPG-5c & 500 & 500 & 250 & 500 \\
\hline 4 & SPG-5d & 100 & 500 & 250 & 500 \\
\hline 5 & SPG-5e & 500 & 500 & 500 & 500 \\
\hline 6 & SPG-5f & 500 & 100 & 100 & 250 \\
\hline 7 & SPG-5g & 500 & 200 & 250 & 500 \\
\hline 8 & SPG-5h & 250 & 250 & 200 & 250 \\
\hline 9 & SPG-5i & 200 & 250 & 500 & 250 \\
\hline 10 & SPG-5j & 200 & 250 & 200 & 500 \\
\hline 11 & SPG-5k & 500 & 250 & 250 & 250 \\
\hline 12 & SPG-5l & 500 & 250 & 250 & 250 \\
\hline 13 & SPG-5m & 100 & 200 & 500 & 125 \\
\hline 14 & SPG-5n & 250 & 200 & 500 & 250 \\
\hline 15 & SPG-5o & 500 & 500 & 250 & 500 \\
\hline 16 & SPG-5p & 250 & 200 & 500 & 200 \\
\hline 17 & SPG-5q & 250 & 500 & 500 & 200 \\
\hline 18 & SPG-5r & 500 & 250 & 250 & 250 \\
\hline 19 & SPG-5s & 250 & 200 & 250 & 500 \\
\hline 20 & SPG-5t & 500 & 500 & 250 & 500 \\
\hline \multicolumn{2}{|c|}{ Gentamycin } & 0.05 & 1 & 0.25 & 0.5 \\
\hline \multicolumn{2}{|c|}{ Ampicilin } & 100 & 100 & 250 & 100 \\
\hline \multicolumn{2}{|c|}{ Chloramphenicol } & 50 & 50 & 50 & 50 \\
\hline \multicolumn{2}{|c|}{ Ciprofloxacin } & 25 & 25 & 50 & 50 \\
\hline \multicolumn{2}{|c|}{ Norfloxacin } & 10 & 10 & 10 & 10 \\
\hline
\end{tabular}


Table 3. Antifungal activity of Compound SPG 3a-t.

\begin{tabular}{|c|c|c|c|c|}
\hline \multirow{2}{*}{ Sr. No. } & \multirow{2}{*}{ Code } & \multicolumn{3}{|c|}{ MIC $(\boldsymbol{\mu g} / \mathbf{m L})$} \\
\cline { 3 - 5 } & & C.albicans & A.niger & A.clavatus \\
\hline $\mathbf{1}$ & SPG-5a & 500 & 500 & $>1000$ \\
\hline $\mathbf{2}$ & SPG-5b & 500 & 500 & 250 \\
\hline $\mathbf{3}$ & SPG-5c & 500 & 500 & 1000 \\
\hline $\mathbf{4}$ & SPG-5d & 500 & 500 & $>1000$ \\
\hline $\mathbf{5}$ & SPG-5e & 500 & 500 & 500 \\
\hline $\mathbf{6}$ & SPG-5f & 200 & 250 & 250 \\
\hline $\mathbf{7}$ & SPG-5g & 500 & 1000 & 500 \\
\hline $\mathbf{8}$ & SPG-5h & 250 & 500 & 500 \\
\hline $\mathbf{9}$ & SPG-5i & 100 & 100 & 100 \\
\hline $\mathbf{1 0}$ & SPG-5j & 500 & 250 & 500 \\
\hline $\mathbf{1 1}$ & SPG-5k & 200 & 500 & 500 \\
\hline $\mathbf{1 2}$ & SPG-5l & 500 & 1000 & 500 \\
\hline $\mathbf{1 3}$ & SPG-5m & 500 & 250 & 250 \\
\hline $\mathbf{1 4}$ & SPG-5n & 250 & 250 & 500 \\
\hline $\mathbf{1 5}$ & SPG-50 & 1000 & 1000 & 500 \\
\hline $\mathbf{1 6}$ & SPG-5p & 250 & 250 & 500 \\
\hline $\mathbf{1 7}$ & SPG-5q & 500 & 250 & 250 \\
\hline $\mathbf{1 8}$ & SPG-5r & 250 & 500 & 250 \\
\hline $\mathbf{1 9}$ & SPG-5s & 500 & 500 & 1000 \\
\hline $\mathbf{2 0}$ & SPG-5t & 500 & 500 & 500 \\
\hline & Nystatin & 100 & 100 & 100 \\
\hline Greseofulvin & 500 & & \\
\hline & & & & 500 \\
\hline
\end{tabular}




\section{RESULT AND DISCUSSION}

Various methodologies have been described for the synthesis of 1,2,4-triazolo[1,5a]pyrimidines. During the course of our ongoing interest on synthesis of various heterocyclic compounds using $\alpha$-oxo ketene dithioacetals, we observed that $\alpha$-oxo ketene dithioacetals are versatile intermediate for the synthesis of triazolopyrimidines.

Thus, to synthesized target molecules, the various $\alpha$-oxo ketene dithioacetals $(2 \mathrm{a}-\mathrm{t})$ were reacted with 5-amino-1,2,4-triazole (2) in the presence sodium methoxide as a base in methanol at reflux temperature to afford 1,2,4-triazolo[1,5-a]pyrimidines (3a-t) (Table 1). Various $\alpha$-oxo ketene dithioacetals (2a-t) was synthesized by reported method ${ }^{20}$ All the synthesized compounds were screened against varieties of bacterial strains (Table 2) such $E$. coli, S. pyogenus, S. aureus, $P$. aeruginosa and fungi strains (Table 3) C. albicans, A. niger, A. clavatus at minimal inhibitory concentration (MIC). Standard drugs like Ampicillin, Chloramphenicol, Nystatin and Greseofulvin were used for the comparison purpose.

\section{CONCLUSION}

In summary, we have described the synthesis of substituted triazolopyrimidine derivatives in moderate yield. The reaction of various $\alpha$-oxo ketene dithioacetals with 5amino-1,2,4-triazole was afforded the triazolopyrimidine derivatives in moderate to good yield in the presence of base. Sodium methoxide was found as an efficient base. All the synthesized compounds were evaluated for their antimicrobial activity. The investigation of antimicrobial and antifungal screening data revealed that all the tested compounds showed moderate to significant activity.

\section{ACKNOWLEDGEMENT}

We are very much thankful to department of chemistry for providing lab facilities and National Facility For Drug Discovery center, saurashtra university for Spectral analysis.

\section{References}

[1] G. Fischer, Adv. Heterocycl. Chem. 57 (1993) 81.

[2] M. Shaban, A. Morgan, Adv. Heterocycl. Chem. 77 (2000) 345.

[3] M. Shaban, A. Morgan, Adv. Heterocycl. Chem., 73 (2000) 131.

[4] M. Shaban, A. Morgan, Adv. Heterocycl. Chem. 75 (2000) 243.

[5] N. Zhang, A. Semiramis, N. Thai, J. Med. Chem. 50 (2007) 319.

[6] L. Havlicek; K. Fuksova; V. Krystof, Bioorg. Med. Chem. 13 (2005) 5399.

[7] X. Zhao; Y. Zhao; S. Guo; H. Song; D. Wang; P. Gong, Molecules 12 (2007) 1136.

[8] L. Iwona, F. Marzena, M. Tadeusz, S. Tadeusz, J. Julia, Dalton Trans. 42 (2013) 6219.

[9] A. Marwaha, J. White, F. El Mazouni, S. Creason, S. Kokkonda, F. Buckner, P. Rathod, J. Med. Chem. 55 (2012) 7425. 
[10] L. Yin; Z. Shuai; L. Zhi-Jun; Z. Hai-Liang, Euro. J. Med. Chem. 64 (2013) 54.

[11] H. Ashraf, Abd El-Wahab Pharmaceuticals 5 (2012) 745.

[12] A. Abdel-Aziem, M. Sayed El-Gendy, A. Abdelhamid, Euro. J. Chem. 3 (2012) 455.

[13] M. Khera, I. Cliffe, T. Mathur, O. Prakash, Bioorg. Med. Chem. Lett. 21 (2011) 2887.

[14] H. Ashour, O. Shaaban, O. Rizk, I. El-Ashmawy, Euro. J. Med. Chem. 62 (2013) 341.

[15] M. Fraley, W. Hoffman, R. Rubino, Bioorg. Med. Chem. Lett. 12 (2002) 2767.

[16] Q. Chen, X. Zhu, Z. Liu, Euro. J. Med. Chem. 43 (2008) 595.

[17] S. Uryu, S. Tokuhiro, T. Murasugi, Brain Research 946 (2002) 298

[18] L. Antonino, A. Ilenia, P. Chiara, M. Annamaria, D. Gaetano, A. Maria, Euro. J. Med. Chem. 62 (2013) 416.

[19] B. Fairfield, C. Andrew, J. Allan, WO2004108136, (2004).

[20] C. Tamilselvan, S. John Joseph, G. Mugunthan, S. Syed Musthaq Ahamed, International Letters of Chemistry, Physics and Astronomy 9 (2014) 93-102.

[21] R. G. Vaghasiya, H. B. Ghodasara, P. R. Vachharajani, V. H. Shah, International Letters of Chemistry, Physics and Astronomy 8 (2014) 30-37.

[22] Piyush B. Vekariya, Jalpa R. Pandya, Vaishali Goswami, Hitendra S. Joshi, International Letters of Chemistry, Physics and Astronomy 7 (2014) 45-52. 